\title{
Pratiques et politiques de mobilité dans un campus universitaire : le cas de l'Université de Lausanne (Suisse)
}

Practices and politics of mobility on a university campus : the case of the University of Lausanne (Switzerland)

\section{Patrick Rérat}

\section{OpenEdition \\ Journals}

Édition électronique

URL : https://journals.openedition.org/developpementdurable/19038

DOI : 10.4000/developpementdurable.19038

ISSN : 1772-9971

Éditeur

Association DD\&T

\section{Référence électronique}

Patrick Rérat, «Pratiques et politiques de mobilité dans un campus universitaire : le cas de l'Université de Lausanne (Suisse) », Développement durable et territoires [En ligne], Vol. 12, n¹ | Mai 2021, mis en ligne le 26 mai 2021, consulté le 28 mai 2021. URL : http://journals.openedition.org/

developpementdurable/19038; DOI : https://doi.org/10.4000/developpementdurable.19038

Ce document a été généré automatiquement le 28 mai 2021.

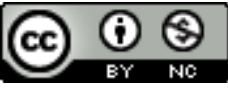

Développement Durable et Territoires est mis à disposition selon les termes de la licence Creative Commons Attribution - Pas d'Utilisation Commerciale 4.0 International. 


\title{
Pratiques et politiques de mobilité dans un campus universitaire : le cas de l'Université de Lausanne (Suisse)
}

\author{
Practices and politics of mobility on a university campus : the case of the \\ University of Lausanne (Switzerland)
}

Patrick Rérat

L'auteur remercie Benoit Frund et Julien Meillard (Durabilité et Campus, Université de Lausanne), Gianluigi Giacomel, Dimitri Marincek et Antonio Martin (Institut de géographie et durabilité, Université de Lausanne) ainsi que Laura Scaperrotta et Boris Wernli (FORS) pour leurs différents apports à cette publication.

1 Les transports représentent un défi central pour les universités qui génèrent quotidiennement des flux importants. Il s'agit de développer leur accessibilité et leur attractivité, mais aussi de réduire leur empreinte écologique (Miralles-Guasch et Domene, 2010; Van den Berg et Russo, 2004). La transition vers une mobilité durable représente un enjeu de taille pour ces institutions, de plus en plus nombreuses à ambitionner de devenir exemplaires en matière de durabilité (Balsas, 2003 ; Cattaneo et al., 2018 ; Delmelle et Delmelle, 2012). La mobilité représente en effet la première source d'émission de gaz à effet de serre pour ces établissements, que ce soit en raison des déplacements pendulaires (traités ici) ou des déplacements ponctuels (colloques, etc.) (Genta et al., 2019).

2 Cet article porte sur l'Université de Lausanne en Suisse, dont plusieurs caractéristiques en font un cas intéressant : elle compte 20000 étudiants et collaborateurs - et ce chiffre est en croissance -, elle couvre la plupart des domaines scientifiques et son campus est localisé en couronne suburbaine et non pas en zone centrale (où il aurait bénéficié d'infrastructures existantes). Une série d'enquêtes par questionnaire et des documents 
de planification dans le domaine des transports et de l'urbanisme permettent d'étudier la mobilité quotidienne induite par cette institution.

3 Les questions de recherche suivantes structurent l'analyse : Quelle part occupent les différents moyens de transport dans les trajets des étudiants et du personnel à destination du campus? Comment ces pratiques varient-elles dans le temps et au sein de la population universitaire? Quelles politiques expliquent et influencent cette répartition modale? Par rapport à la littérature existante, cette contribution se distingue par la prise en compte simultanée de la demande (les choix modaux des étudiants comme du personnel) et de l'offre de transport (les politiques de mobilité ayant eu une influence sur le campus), mais aussi par la période couverte (13 enquêtes annuelles consécutives). Elle participe ainsi aux recherches sur la mobilité sur les campus universitaires et sur les leviers pour les transformer en communautés durables.

L'article commence par un état de la question de l'étude de la mobilité (pratiques et politiques) dans le cas des campus. Dans un deuxième temps, la démarche de recherche est expliquée. Sont ensuite présentés les résultats relatifs à la demande et à l'offre de mobilité. La conclusion revient sur les principaux enseignements et sur les enjeux auxquels sont confrontées les universités en matière de mobilité.

\section{Analyser la mobilité sur un campus universitaire}

5 Ces dernières années, un corpus de recherches sur la mobilité en milieu universitaire s'est développé. Au-delà de la facilité d'accès au terrain, plusieurs raisons expliquent l'intérêt des chercheurs. Les universités figurent parmi les plus grands générateurs et attracteurs de flux de navetteurs (Rotaris et Danielis, 2015). Leur localisation, souvent suburbaine, rend les enjeux de mobilité plus aigus (Miralles-Guasch et Domene, 2010). Les campus accueillent une population variée du point de vue de l'âge et du statut socio-économique (quand bien même celle-ci ne reflète pas la population générale). Ils apparaissent comme des lieux privilégiés pour communiquer et concrétiser la durabilité, et comme des laboratoires afin de tester et mettre en œuvre des stratégies alternatives de transport (Balsas, 2003). Les tendances observées peuvent se révéler par ailleurs pertinentes pour d'autres types de campus (parcs d'activités, complexes de bureaux, hôpitaux, etc.) (Tolley, 1996).

6 La période des études est en outre propice à l'apprentissage de différentes pratiques. Alamel a par exemple montré que les étudiants sont des « apprentis résidents » dans le domaine de la consommation d'énergie domestique, de la gestion d'un budget, etc. (Alamel, 2015). Un parallèle peut être tiré avec l'apprentissage de pratiques de mobilité durable qui, si elles se construisent dans des conditions favorables, pourraient perdurer dans le parcours de vie (Delmelle et Delmelle, 2012). Les campus universitaires représentent des microcosmes, des lieux où les normes et les comportements sont façonnés et, par conséquent, un contexte idéal pour explorer et tester des politiques et des mesures visant à réduire la dépendance automobile (Balsas, 2003). Cet effet potentiel serait d'autant plus intéressant que les étudiants sont amenés à occuper des positions d'influence dans les administrations publiques, les entreprises et autres organisations (Tolley, 1996, 214). 


\subsection{Les choix modaux à destination des campus}

7 Le choix modal se définit comme la décision de recourir à un moyen de transport pour réaliser un déplacement. Il est souvent le résultat d'un choix complexe qui se déroule de manière consciente ou non et qui peut être contraint par des circonstances objectives et subjectives (Courel et Deguitre, 2020 ; De Witte et al., 2013).

8 Sur la base d'une revue de la littérature, De Witte et al. (2013) identifient une liste, non exhaustive, de 26 déterminants répartis en quatre catégories: les variables sociodémographiques (âge, genre, formation, revenu, ménage, etc.), les variables spatiales (densité, mixité fonctionnelle, proximité des infrastructures et des services, fréquence des transports publics, conditions de stationnement, etc.), les caractéristiques du déplacement (motif, distance, durée, coût, etc.) et des dimensions sociopsychologiques (expériences, habitudes, valeurs, perceptions, etc.). Les variables sociodémographiques et spatiales déterminent les possibilités en termes de mobilité alors que les dimensions psychosociologiques influencent la manière dont ces possibilités sont appropriées ou mises en œuvre (ibid. 331). Les dimensions psychosociologiques renvoient aux quatre logiques d'action des pratiques modales que Kaufmann identifie en s'inspirant des travaux de Max Weber (Weber, 1922): la rationalité économique (calcul comparatif), la rationalité en valeurs (préférence), la rationalité perceptive (ressenti) et les habitudes (réflexe) (Kaufmann, 2003).

9 Dans le cas spécifique des campus, les recherches identifient souvent une forte dépendance à la voiture (Cattaneo et al., 2018; Choplin et Delage, 2011; dell'olio et al., 2019 ; Melia et Clark, 2018 ; Tolley, 1996), qui est d'autant plus prégnante lorsqu'ils sont éloignés du centre-ville (Vale et al., 2018). Des divergences apparaissent au sein des communautés universitaires. Les étudiants, en raison de leur âge et de leurs moyens financiers limités, possèdent moins souvent un permis de conduire ou une voiture. Ils recourent ainsi moins que les salariés à l'automobile. Parmi ces derniers, les enseignants-chercheurs utilisent moins la voiture que les membres de l'administration, en raison notamment de localisations résidentielles plus urbaines (Miralles-Guasch et Domene, 2010). Les étudiants recourent davantage aux transports en commun, mais utilisent aussi le vélo en raison du faible coût et de leurs conditions physiques (Shannon et al., 2006). D'autres divergences ont été relevées selon les facultés. Il a été observé que les étudiants ont des attitudes et des traits de personnalité différents selon les facultés, et que cela a un impact sur les pratiques de mobilité (Cattaneo et al., 2018; Kim et al., 2016). À titre d'illustration, les étudiants en génie civil qui suivent des cours sur les questions environnementales ont une plus grande conscience de ces problématiques, ce qui influence à son tour leur attitude quant aux moyens de transport (Ibid.).

10 Les recherches s'intéressent également au potentiel de report modal des transports individuels motorisés vers les transports en commun et les mobilités actives que sont la marche et le vélo (Rotaris et Danielis, 2015). Certaines montrent que les comportements ne correspondent pas toujours aux préférences des usagers et que ces derniers sont contraints mais aussi satisfaits à des niveaux très variables dans leurs pratiques de mobilité (Miralles-Guasch et Domene, 2010). Des études portent sur les motivations et freins à l'adoption de modes actifs (Shannon et al., 2006). La diversité des pendulaires est également mise en exergue par l'identification de catégories selon l'attitude relative aux alternatives à la voiture et aux mesures qui pourraient les inciter à modifier leurs 
pratiques (Fürst, 2014). Ce dernier point ouvre la discussion sur l'offre de transport des campus et les politiques afférentes.

\subsection{Les politiques de mobilité des campus universitaires}

11 Sur la base de la littérature concernant les campus universitaires, mais aussi plus généralement les politiques de mobilité, nous proposons de distinguer quatre grandes figures ou types de mesure et de les dénommer respectivement "campus relié", " campus régulé », « campus intégré » et " campus connecté ». Ces figures résument les différentes politiques de mobilité pouvant influencer la mobilité à destination d'un campus. Il est à préciser que ces mesures ne sont pas du seul ressort des universités. En effet, si on les compare parfois à de petites villes, leurs prérogatives sont limitées et les différents pouvoirs publics constituent des acteurs centraux.

Le campus relié renvoie à une politique de l'offre et à la construction d'infrastructures dans le but de répondre à la demande. Pour une université, le besoin en infrastructures dépend notamment de sa localisation. De nombreux campus, en raison de leur localisation suburbaine, de la proximité d'axes routiers et de la présence abondante de parkings, se caractérisent par une prédominance de l'automobile (Cattaneo et al., 2018 ; dell'Olio et al., 2019; Melia et Clark, 2018; Tolley, 1996). La promotion de moyens alternatifs se heurte alors au manque d'aménagements dédiés, à l'absence de reconnaissance des mobilités actives et à la durée plus longue des déplacements en transports en commun (Miralles-Guasch et Domene, 2010).

Le campus régulé renvoie à la gestion de la demande («mobility management») et à des mesures dites douces (dans le sens de non infrastructurelles). La gestion de la demande de mobilité peut être définie comme un ensemble de stratégies d'aménagement (ex. : la modération du trafic) et de mesures d'incitation ou de dissuasion visant à modifier les comportements de mobilité et promouvoir un report modal des transports individuels motorisés vers les autres modes (Balsas, 2003 ; Miralles-Guasch et Domene, 2010).

Plusieurs études ont montré l'importance de la régulation du stationnement, en particulier dans le cas des campus. Le stationnement gratuit ou bon marché est vu comme une subvention pour les étudiants et le personnel recourant à la voiture sur le campus, alors que ceux qui utilisent le vélo, la marche ou les transports publics sont rarement récompensés financièrement (Brown et al., 2001). Il rend également l'usage de la voiture attractif de manière générale, mais aussi pour les trajets de courte distance et les déplacements spontanés, notamment pendant la saison froide (Delmelle et Delmelle, 2012). Des recherches ont analysé les changements de comportement induits par une tarification ou une restriction du stationnement sur les campus (dell'olio et al., 2019; Melia et Clark, 2018). D'autres mesures portent sur la subvention des abonnements de transports en commun (Brown et al., la mise en place de services de covoiturage et d'autopartage, l'achat des véhicules moins polluants pour la flotte des universités, la flexibilisation du temps de travail ou encore des campagnes de sensibilisation (Balsas, 2003 ; Miralles-Guasch et Domene, 2010).

15 Les politiques des transports, qu'elles touchent l'offre ou la demande, ne constituent qu'une manière d'influencer la mobilité. Il existe un potentiel considérable d'influencer les déplacements au travers de politiques qui se trouvent en dehors du domaine des transports comme le développement territorial et l'urbanisme (Stead et Banister, 2001). La figure du campus intégré renvoie tout d'abord à la localisation des campus qui a une 
grande influence sur les pratiques de mobilité (Vale et al., 2018). Pour les universités implantées au cœur des agglomérations, les logements, commerces, services et aménités sont souvent situés à proximité (Delmelle et Delmelle, 2012). Les campus périphériques font souvent la part belle à l'accessibilité en voiture (voir ci-dessus). Le modèle nord-américain du campus, qui suit les principes de l'urbanisme néotraditionnel, combine une variété de fonctions accessibles à pied (Balsas, 2003).

Outre le choix de la localisation, des débats portent sur les formes urbaines et leur adéquation avec les principes de durabilité (Dempsey et Jenks, 2010 ; Rérat, 2012 ; Stead et Banister, 2001). Le modèle de la ville compacte doit permettre de réduire la dépendance automobile, de rendre les transports en commun attractifs et d'offrir des environnements favorables à la marche et au vélo. Ce modèle renvoie à des principes tels que la coordination entre urbanisation et transport, des densités accrues et une mixité des fonctions. Il fait également référence aux différents rôles que les universités peuvent endosser dans la production urbaine (constructeur, promoteur, etc.) (Bourdin et Campagnac, $2014 ; \mathrm{Vu}, 2014)$.

Dernière figure, le campus connecté est lié à la numérisation qui ouvre de nouvelles perspectives dans le domaine du télétravail et de l'enseignement à distance. Un enjeu clé ici est le degré auquel télécommunications et déplacements sont des substituts ou des compléments dans la pratique du télétravail (Lachapelle et al., 2018). Une partie du temps économisé pourrait être réinvestie dans d'autres déplacements à motif non professionnel et influencer les choix de localisation du lieu de domicile et du lieu de travail (Ravalet et Rérat, 2019).

Cette grille de lecture a été élaborée afin d'analyser les mesures prises pour gérer les flux de transport à destination du campus de l'Université de Lausanne et sera également utilisée afin d'interpréter l'évolution des pratiques de mobilité des étudiants et du personnel.

\section{Démarche de recherche}

\subsection{Le campus de l'Université de Lausanne}

Dans les années 1960, l'Université de Lausanne (Unil), alors implantée au cœur de la ville, connait un essor important qui incite les autorités à chercher un nouveau site pour son développement. Elles optent pour Dorigny, un site encore essentiellement rural à l'ouest de Lausanne, et qui accueille un premier bâtiment en 1970. Depuis, une vingtaine d'autres ont été construits pour les activités académiques, mais sans accueillir de logements. Ils sont tantôt dispersés, tantôt concentrés, avec des passages par de vastes espaces verts selon un concept de "pavillons dans un parc " (Maillard, 2013) (Figure 1). L'Unil compte 20000 étudiants et collaborateurs (dont $90 \%$ sont actifs sur le campus de Dorigny) et couvre tous les grands domaines scientifiques à l'exception des sciences de l'ingénieur. Le concept de circulation interne au campus est fondé sur l'élimination des routes - les parkings étant situés à la marge - afin que les personnes se déplacent essentiellement à pied. 


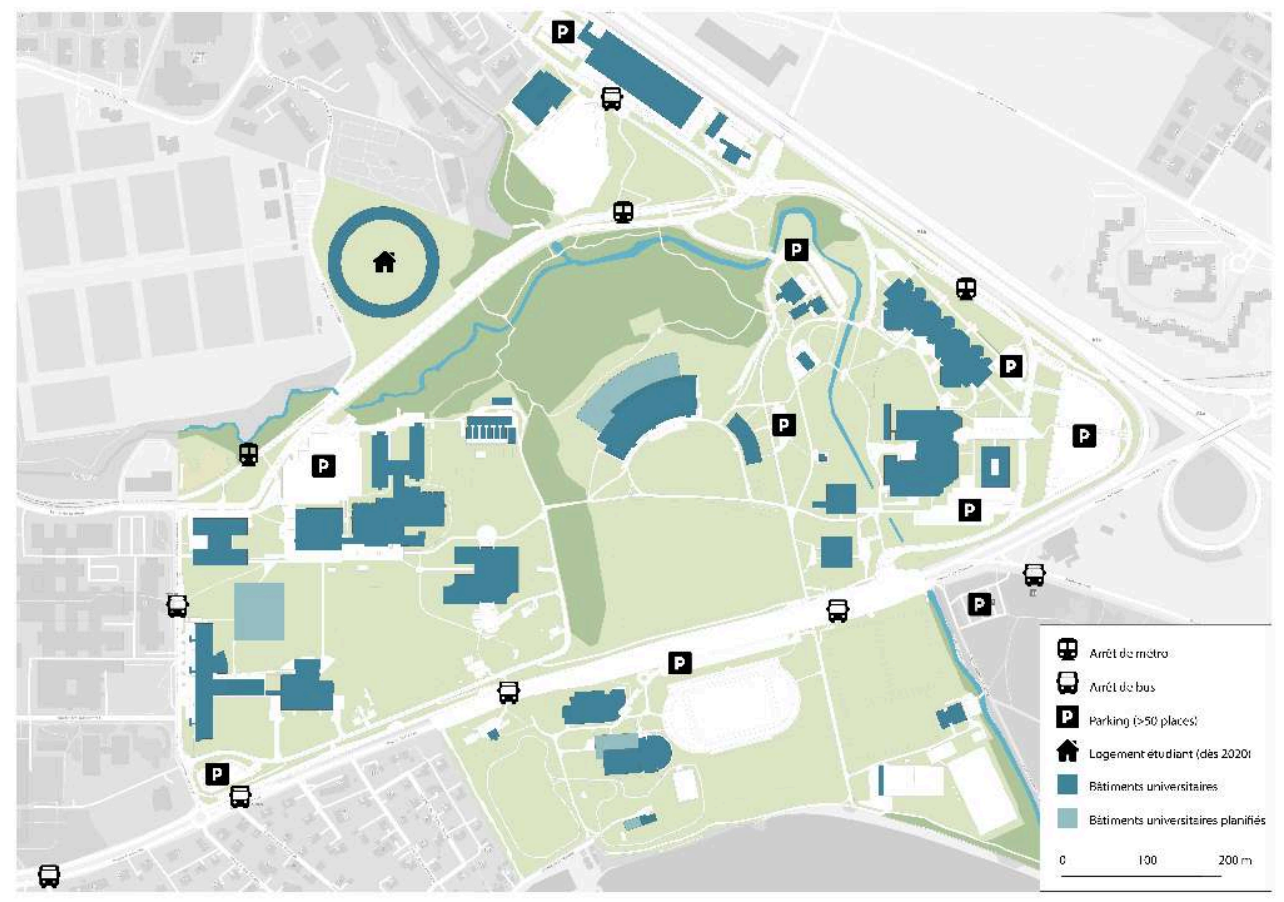

Source : Université de Lausanne, réalisé par Dimitri Marincek

Le campus se situe à $5 \mathrm{~km}$ de la gare de Lausanne et $2 \mathrm{~km}$ de celle de Renens, qui sont les plus fréquentées de Suisse romande après Genève. Si la fréquentation de la gare de Lausanne correspond à sa population (deuxième ville romande avec 145000 habitants), celle de Renens (21 000 habitants) s'explique par la proximité de l'Unil et de l'École polytechnique fédérale de Lausanne. Des liaisons ferroviaires (au moins une par heure) relient Lausanne aux autres grandes villes suisses alors que Renens est très bien connectée à l'ensemble de l'Arc lémanique ${ }^{1}$. Un métro dessert ces gares et le campus, de même que plusieurs lignes de bus (les arrêts sont représentés sur la figure 1). Par ailleurs, la ville de Lausanne se caractérise par des pentes importantes et un manque d'infrastructures cyclables, si bien qu'elle fait partie des villes suisses les moins bien évaluées par les cyclistes en termes de sécurité (Rérat et al., 2019).

Le contexte dans lequel s'insère le campus a considérablement évolué et s'est progressivement urbanisé (Coen et Lambelet, 2011). Cette région suburbaine, l'Ouest lausannois, connaît l'une des plus fortes croissances démographiques et économiques de Suisse. Le campus, autrefois isolé, est désormais entouré par l'urbanisation, quand bien même, à une échelle plus fine, des coupures physiques persistent, à cause notamment des infrastructures de transport.

\subsection{Méthodologie}

La demande de mobilité est abordée grâce à une enquête par questionnaire réalisée chaque année entre 2005 et 2017 au printemps par le Laboratoire Intermodalités, Transports et Planification (Litep) de l'École polytechnique fédérale de Lausanne et dont les fichiers bruts ont été mis à disposition pour cette recherche par l'Université de Lausanne. Cette enquête est adressée électroniquement à l'ensemble des étudiants et 
du personnel de l'Unil, dont les effectifs sont passés de 12000 à 18000 pendant la période étudiée.

Le questionnaire demande aux répondants de renseigner la manière dont ils effectuent habituellement les déplacements entre le domicile et le campus. Il aborde plus précisément leurs caractéristiques spatiales (communes d'origine et bâtiments de destination), temporelles (heures d'arrivée et de départ sur le campus, fréquence des trajets) et modales (moyens de transport) ${ }^{2}$. Des informations relatives au profil des personnes interrogées sont obtenues en croisant le questionnaire et la base de données de l'Unil tout en garantissant l'anonymat.

Le taux de réponse oscille autour de $25 \%$. Un redressement de l'échantillon corrige les taux de réponse variables selon les groupes d'usagers. Les critères de pondération sont l'âge, le genre et le statut (personnel académique, personnel administratif et technique, étudiants). Le questionnaire est resté quasiment le même entre 2005 et 2017, et a fait l'objet d'une refonte en 2018. La comparabilité est assurée pour la plupart des indicateurs sans être parfaite, si bien que les dernières éditions ne sont pas intégrées à l'analyse.

L'analyse de la demande porte sur le recours aux différents moyens de transport à un niveau agrégé (4.1), puis s'effectue en fonction de caractéristiques susceptibles d'influencer le choix modal (4.2). Dans cette étape, la variable à expliquer est le recours à l'une de ces trois catégories : transports publics; mobilités actives (marche, vélo et autres) et véhicules individuels motorisés (à deux ou quatre roues) ${ }^{3}$. Les variables explicatives testées sont:

- le sexe ;

- l'âge (jusqu'à 25 ans $^{4}, 26-35$ ans, 36-50 ans, 51 ans et plus);

- la distance à vol d'oiseau entre le domicile et le campus ${ }^{5}$;

- la fréquence hebdomadaire des déplacements ;

- le type de commune de résidence selon des critères morphologiques (densité, taille démographique) et fonctionnels (flux pendulaires, spécialisation économique) (OFS, 2014). Cette typologie distingue des centres urbains grands, moyens et petits. Pour les deux premiers sont définies également des couronnes, et pour les grands centres sont identifiés des centres secondaires. Trois types complètent la liste : les communes rurales périurbaines, agricoles et touristiques;

- la faculté (théologie et de sciences des religions; droit, sciences criminelles et administration publique; lettres; sciences sociales et politiques; hautes études commerciales; biologie et médecine; géosciences et environnement) ainsi que des institutions associées (fondations, centres de recherche) et l'administration centrale ;

- le statut qui distingue les étudiants ${ }^{6}(73,7 \%$ des effectifs en 2017), le personnel administratif et technique $(11,8 \%)$ et le personnel académique comprenant les enseignants et chercheurs (professeurs, assistants-doctorants, post-doctorants, etc.);

- l'année de l'enquête qui est introduite dans le modèle comme une variable continue étant donné la régularité de la tendance entre 2005 et $2017^{7}$.

Des régressions logistiques binaires multivariées mesurent l'association entre l'utilisation d'un moyen de transport et les facteurs susceptibles de l'influencer. Elles tiennent compte simultanément de l'ensemble des variables explicatives et ainsi mesurent l'effet propre de chacune d'entre elles («toutes choses égales par ailleurs »). Cet effet est exprimé en termes de rapport de probabilité (odd ratio). S'il est supérieur (inférieur) à 1, la modalité augmente (diminue) la propension à avoir le permis par 
rapport à la modalité de référence ${ }^{8}$. Des tests déterminent si cet effet est statistiquement significatif.

Si cette approche permet d'appréhender les grandes caractéristiques de la demande de mobilité à destination du campus, elle comporte certaines limites. Premièrement, elle tient compte de l'intermodalité (combiner plusieurs modes lors d'un trajet même si les parties réalisées à pied ne sont pas comptabilisées), mais pas de la multimodalité (changer de modes de transport pour se rendre au campus selon le jour, la saison, etc.). Deuxièmement, les résultats portent sur un jour théorique, car toutes les personnes ne se rendent pas quotidiennement sur le campus, y compris en période de cours (temps partiel, télétravail, etc. $\left.{ }^{9}\right)$. Troisièmement, l'enquête aborde des éléments factuels et n'interroge pas les individus sur les raisons de leur choix (aspirations, contraintes, etc.). Finalement, la base de données de l'Unil ne comporte pas le type de ménage (ou la structure familiale) et le revenu n'est que très imparfaitement mesuré par le statut.

La deuxième partie de l'article porte sur l'offre de mobilité caractérisant le campus. Elle se base sur des documents de planification et sur l'histoire du campus du point de vue de l'urbanisme et des transports (Jarne et al., 2011 ; Maillard, 2013) ainsi que sur le tracé des lignes et les horaires des transports publics. Cette démarche permet d'identifier les grands types de mesures qui ont influencé les pratiques de mobilité à destination du campus.

\section{Pratiques de mobilité pour se rendre sur le campus}

\section{1. Évolution des parts modales}

La répartition entre moyens de transport est exprimée en pourcentages (Figure 2) et en valeurs absolues (Figure 3). En 2017, 60,9\% des répondants se déplacent en transports publics uniquement. Suivent à distance la voiture $(16,1 \%)$ et le vélo $(8,8 \%)$. La combinaison de moyens représente une proportion de $8,7 \%$. La marche $(2,4 \%)$ et les deux-roues motorisés $(2,9 \%)$ enregistrent des parts très faibles, de même que les autres moyens $(0,2 \%)$ tels que la trottinette, le skateboard, etc.

Figure 2. Parts des moyens de transport (2005-2017)

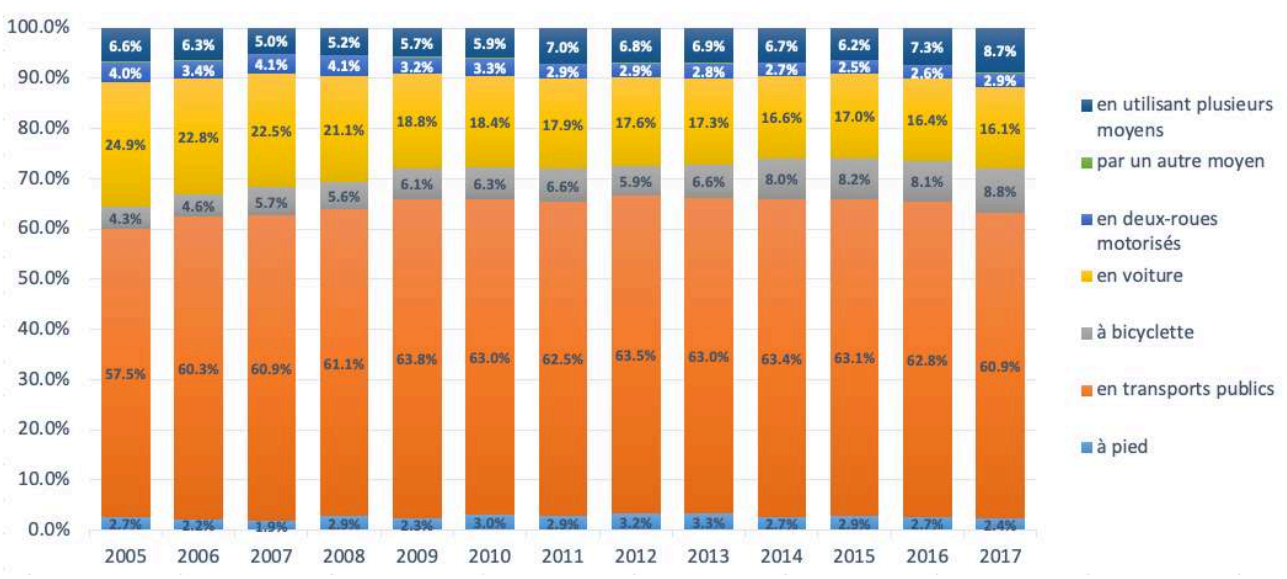


30 La part des transports publics augmente jusqu'en 2009, se stabilise, puis connait un léger recul. Le nombre théorique d'usagers quotidiens, dans l'hypothèse où l'ensemble d'entre eux étaient présents le même jour sur le campus, augmente quant à lui fortement de 6000 à 10000 . Dans $68 \%$ des déplacements en transports publics, au moins deux types sont combinés (train et métro par exemple), alors que $26,2 \%$ d'entre eux se font uniquement en métro et $4,8 \%$ en bus. Les trois quarts des déplacements combinés comptent au moins un trajet en train et près de $90 \%$ du total des déplacements en transports publics comprennent au moins une étape en métro (combinaison ou métro seul). À cette catégorie s'ajoutent une majorité des personnes recourant à plusieurs modes de transport lors de leur trajet et dont le nombre a doublé en 12 ans. L'intermodalité correspond le plus souvent à l'usage d'un deux-roues (motorisé ou non) pour se rendre à une gare (le «premier kilomètre »), puis à l'usage du métro. Cette tendance est un indicateur d'une aire d'attraction de l'Unil qui s'étend (voir 5.1).

31 La part de l'automobile enregistre une réduction significative de $25 \%$ à $16 \%$. En valeurs absolues, une stabilisation autour de 2500 personnes est constatée, un chiffre qui est à mettre en regard de la politique de stationnement (voir 5.2). Neuf usagers sur dix sont des conducteurs seuls et les $10 \%$ restants se partagent à parts égales entre les personnes qui se font déposer sur le campus et les membres de la communauté universitaire pratiquant le covoiturage. Ce dernier est plus fréquent parmi les étudiants (15\%), mais reste globalement stable durant la période considérée.

Une croissance importante de la part du vélo est observée (de 4,3 \% en 2005 à 8,8 \% en 2017), soit un triplement en termes d'effectif (de 500 à 1500 ). Les cyclistes utilisent à $91,2 \%$ un vélo mécanique et $8,8 \%$ recourent à un vélo à assistance électrique. Comme la proportion de personnes vivant dans un rayon de $2 \mathrm{~km}$ du campus n'a que légèrement augmenté (de 10 à $11 \%$ ) et que celle des habitants entre 2 et $5 \mathrm{~km}$ a baissé de manière importante (de 41 à $33 \%$ ), cette évolution est avant tout le résultat d'un regain d'attractivité du vélo et non la conséquence d'une modification des localisations résidentielles. En contraste, la part et le nombre d'usagers des deux-roues motorisés et de la marche varient peu.

Figure 3. Nombre théorique quotidien d'usagers des moyens de transport (2005-2017)

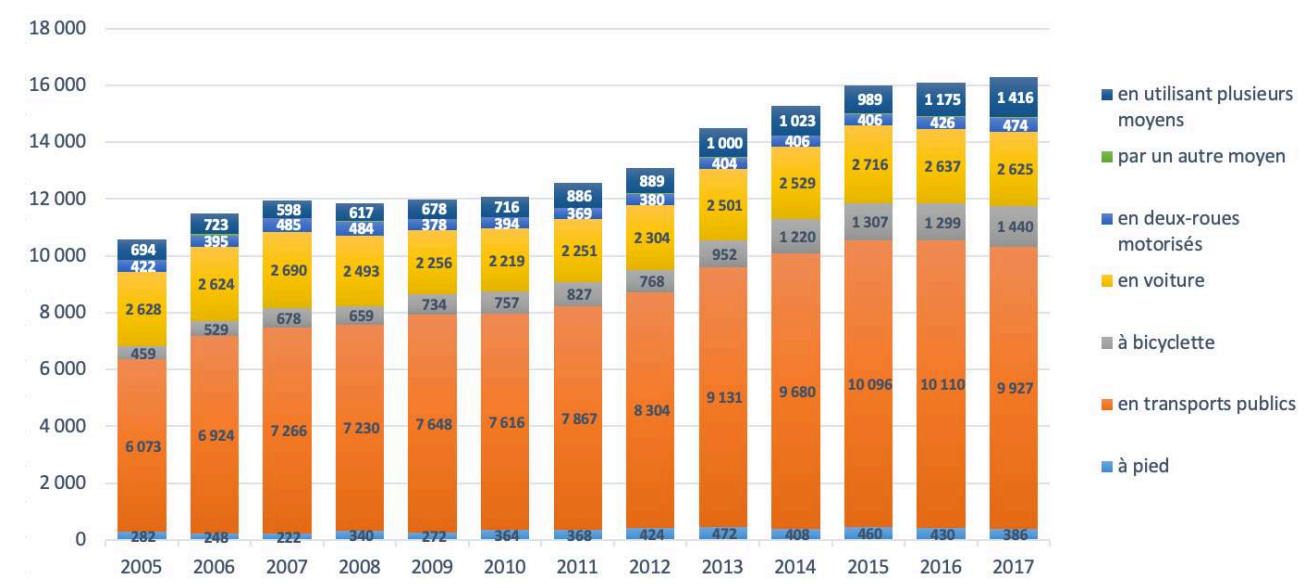




\subsection{Des pratiques différenciées au sein de la communauté universitaire}

Derrière les chiffres agrégés, des variations conséquentes sont observées au sein de la communauté universitaire. Des régressions logistiques ont été appliquées afin d'identifier les différences dans l'usage des transports publics, des mobilités actives et des véhicules individuels motorisés (Tableau 1 ; le tableau des fréquences se trouve en annexe).

Tableau 1. Recours aux différents moyens de transport en fonction du profil des répondants (régressions logistiques binaires)

\begin{tabular}{|c|c|c|c|c|c|c|c|}
\hline \multirow[t]{2}{*}{ Variables } & \multirow[t]{2}{*}{ Modalités } & \multicolumn{2}{|c|}{$\begin{array}{l}\text { Transports } \\
\text { publics }\end{array}$} & \multicolumn{2}{|c|}{$\begin{array}{l}\text { Mobilités } \\
\text { actives }\end{array}$} & \multicolumn{2}{|c|}{$\begin{array}{l}\text { Transports } \\
\text { individuels } \\
\text { motorisés }\end{array}$} \\
\hline & & $\operatorname{Exp}(B)$ & Sig. & $\operatorname{Exp}(B)$ & Sig. & $\operatorname{Exp}(B)$ & Sig. \\
\hline \multirow[t]{2}{*}{ Sexe } & Féminin (réf) & & & & & & \\
\hline & Masculin & 0,798 & $* * *$ & 1,725 & $* * *$ & 1,000 & ns \\
\hline \multirow[t]{4}{*}{ Âge } & Jusqu'à 25 ans (réf) & & & & & & \\
\hline & $26-35$ ans & 0,526 & $* * *$ & 1,389 & $* * *$ & 1,986 & $* * *$ \\
\hline & $36-50$ ans & 0,285 & $* * *$ & 1,202 & $*$ & 4,106 & $* * *$ \\
\hline & 51 ans et plus & 0,196 & $* * *$ & 0,973 & ns & 6,253 & $* * *$ \\
\hline \multirow[t]{3}{*}{ Statut } & Étudiant (réf) & & & & & & \\
\hline & $\begin{array}{l}\text { Personnel administratif et } \\
\text { technique }\end{array}$ & 0,430 & $* * *$ & 0,535 & $* * *$ & 3,354 & $* * *$ \\
\hline & Personnel académique & 0,737 & $* * *$ & 1,217 & $* *$ & 1,364 & $* * *$ \\
\hline \multirow[t]{9}{*}{ Faculté } & Administration centrale (réf) & & & & & & \\
\hline & Hautes études commerciales & 0,593 & $* * *$ & 0,503 & $* * *$ & 2,696 & $* * *$ \\
\hline & Biologie et médecine & 0,856 & ns & 1,101 & ns & 1,034 & ns \\
\hline & $\begin{array}{l}\text { Droit, sciences criminelles et } \\
\text { administration publique }\end{array}$ & 0,719 & $* * *$ & 0,537 & $* * *$ & 2,027 & $* * *$ \\
\hline & $\begin{array}{l}\text { Théologie et sciences des } \\
\text { religions }\end{array}$ & 1,375 & * & 0,725 & ns & 0,780 & ns \\
\hline & $\begin{array}{l}\text { Géosciences et } \\
\text { environnement }\end{array}$ & 0,710 & $* * *$ & 1,671 & $* * *$ & 0,887 & ns \\
\hline & Lettres & 1,184 & $* * *$ & 0,751 & ns & 0,893 & ns \\
\hline & Sciences sociales et politiques & 0,857 & ns & 0,821 & ns & 1,304 & $* * *$ \\
\hline & Institutions associées & 1,420 & $* * *$ & 0,974 & ns & 0,713 & $* * *$ \\
\hline $\begin{array}{l}\text { Type de } \\
\text { commune }\end{array}$ & Grands centres (réf) & & & & & & \\
\hline
\end{tabular}




\begin{tabular}{|c|c|c|c|c|c|c|c|}
\hline & $\begin{array}{l}\text { Centres secondaires des } \\
\text { grands centres }\end{array}$ & 0,339 & $* * *$ & 3,310 & $* * *$ & 1,606 & *** \\
\hline & $\begin{array}{l}\text { Couronnes des grands } \\
\text { centres }\end{array}$ & 0,276 & $* * *$ & 2,314 & $* * *$ & 3,106 & $* * *$ \\
\hline & Centres moyens & 0,622 & $* * *$ & 0,940 & ns & 1,412 & $* * *$ \\
\hline & $\begin{array}{l}\text { Couronnes des centres } \\
\text { moyens }\end{array}$ & 0,261 & $* * *$ & 0,952 & ns & 3,347 & $* * *$ \\
\hline & Petits centres & 0,176 & $* * *$ & 1,146 & ns & 5,238 & *** \\
\hline & $\begin{array}{ll}\text { Communes } & \text { rurales } \\
\text { périurbaines } & \end{array}$ & 0,144 & $* * *$ & 1,175 & ns & 6,379 & $* * *$ \\
\hline & Communes agricoles & 0,107 & $* * *$ & 0,956 & ns & 8,920 & $* * *$ \\
\hline & Communes touristiques & 0,273 & $* * *$ & 1,499 & ns & 3,528 & *** \\
\hline \multirow[t]{3}{*}{ Distance } & Moins de 5 km (réf) & & & & & & \\
\hline & $5-15 \mathrm{~km}$ & 1,102 & $*$ & 0,167 & $* * *$ & 2,588 & *** \\
\hline & Plus de $15 \mathrm{~km}$ & 3,149 & $* * *$ & 0,040 & $* * *$ & 1,039 & ns \\
\hline Fréquence & 1 à 7 jours par semaine & 1,066 & $* * *$ & 0,967 & ns & 0,938 & $* * *$ \\
\hline $\begin{array}{l}\text { Année } \\
\text { d'enquête }\end{array}$ & De 2005 à 2017 & 1,035 & $* * *$ & 1,060 & $* * *$ & 0,925 & $* * *$ \\
\hline
\end{tabular}

Notes : $n$ s $=$ non significatif; ${ }^{*}=p<.05 ; * \star=p<.01 ; * \star \star * p<.001$

Indicateur de fit des modèles - R2 de Nagelkerke : 0,233;0,257;0,306

Le recours aux transports individuels motorisés - avant tout la voiture - est, toutes choses égales par ailleurs, identique entre hommes et femmes. Il est moins fréquent parmi les jeunes et augmente au fil du parcours de vie. Cet accès différencié à l'automobile s'explique par un effet de revenu (qui offre une plus grande latitude dans les choix modaux), mais aussi un double effet d'âge et de génération (les jeunes n'ont pas encore forcément leur permis de conduire et ils semblent avoir moins été socialisés à la voiture) (Rérat et al., 2016 ; Rérat et Haldimann, 2020). Les facultés d'économie et de droit, et dans une moindre mesure des sciences sociales et politiques, utilisent davantage la voiture toutes choses égales par ailleurs. Ces différences qui apparaissent quand bien même ces facultés partagent le même campus semblent révéler l'importance de l'image des moyens de transport et des valeurs qui leur sont associées.

Un fort effet du contexte territorial se manifeste : l'usage de la voiture augmente avec l'éloignement des centres urbains et atteint son maximum dans les communes agricoles et périurbaines. Les distances pour lesquelles les transports individuels motorisés sont particulièrement concurrentiels se situent entre 5 et $15 \mathrm{~km}$. Finalement, la part de la voiture diminue, toutes choses égales par ailleurs, au fil des enquêtes. Comme la méthode utilisée permet de contrôler les effets de structure, cette tendance baissière ne s'explique pas par des changements dans la composition de la communauté universitaire (davantage d'étudiants que de collaborateurs par exemple), mais bien par des changements de pratiques.

L'usage des transports publics se renforce avec le temps. Il est plus fréquent chez les femmes. À l'inverse des transports individuels motorisés, il est plus élevé parmi les 
moins de 25 ans et décline avec l'âge. Le statut est aussi très discriminant: les plus assidus sont les étudiants, suivis du personnel académique, le personnel administratif et technique fermant la marche. Cette différence au sein du personnel pourrait s'expliquer par la valorisation du temps de trajet en transports publics pour le travail qui concernerait davantage les enseignants-chercheurs. La faculté de théologie, les lettres et les institutions associées à l'Unil se distinguent par un recours supérieur à la moyenne.

Un usage très intense caractérise les grands centres à l'instar de Lausanne, mais aussi d'autres villes reliées aux gares de Lausanne et de Renens. La distance domicile-campus accroît également cet usage, le train étant très compétitif sur de longues distances en termes de vitesse, de confort et de valorisation du temps de trajet. La fréquence hebdomadaire des déplacements augmente l'usage des transports publics (alors qu'elle diminue le recours aux véhicules motorisés).

Les mobilités actives - dominées par le vélo - sont davantage le fait des hommes, une observation fréquente dans des contextes où le vélo occupe une faible part modale (Rérat et al., 2019). L'âge n'a pas un effet linéaire : les mobilités actives sont surtout le fait des 26-35 ans et, dans une moindre mesure des 36-50 ans, alors que les plus âgés et les plus jeunes y recourent moins. Plusieurs hypothèses peuvent être avancées: condition physique moins bonne des plus âgés, problème de stationnement à domicile pour les étudiants qui vivent davantage dans des immeubles anciens, manque d'habitude ou image moins favorable chez ces deux tranches d'âge, plus grande sensibilité des personnes d'âge moyen sur la question de l'opportunité de faire de l'exercice et des bénéfices en termes de santé (ibid.), etc. Les facultés d'économie et de droit se distinguent par un recours bien plus faible que la moyenne aux mobilités actives. À l'inverse, la faculté des géosciences et de l'environnement en fait bien plus usage, ce qui s'explique certainement par une sensibilité environnementale plus aiguisée de ses membres.

La distance diminue très fortement le recours aux mobilités actives en raison de l'effort physique requis. Les types de communes où leur part est la plus élevée reflètent le contexte territorial du campus (une couronne de grand centre à proximité du centre secondaire qu'est Renens). Enfin, la prise en compte de l'année d'enquête dans le modèle montre une tendance à l'augmentation toutes choses égales par ailleurs.

Dans l'ensemble, les pratiques de mobilité divergent au sein de la communauté universitaire quand bien même la destination, le campus, est identique. Le recours aux différents moyens de transport s'explique par l'articulation d'effets de genre, d'âge, de revenu, de contexte territorial (offre disponible et distance) et de valeurs (comme le reflètent notamment les différences entre facultés).

\section{Politiques de la mobilité concernant le campus de l'Unil}

41 La mobilité constitue un enjeu de taille pour l'Unil dès l'inauguration de son campus. Il s'agit d'assurer l'accessibilité de ce site suburbain, puis de faire face à la croissance du nombre d'usagers. À ces enjeux s'ajoute la volonté de promouvoir des pratiques de mobilité durables. Les mesures pour répondre à ces enjeux reprennent les figures 
proposées dans la partie théorique, soit le campus relié, régulé, intégré et connecté, et que nous appliquons au cas de l'Unil.

\subsection{Le campus relié}

42 Le principe du campus relié consiste en un développement de l'offre pour assurer l'accessibilité du campus et répondre à la demande de déplacement. Le campus bénéficie à son inauguration d'une très bonne accessibilité routière, y compris grâce à la proximité de la première autoroute de Suisse. La prédominance de la voiture se traduit par l'aménagement de vastes aires de stationnement, longtemps restées libres d'accès et gratuites. L'offre de transports publics prend la forme d'un transport scolaire classique : des navettes relient le campus à Lausanne par six bus journaliers circulant matin, midi et soir. Cette offre se révèle vite inadaptée et des lignes de bus à service régulier sont mises en exploitation.

43 Une infrastructure de transport en commun à haute capacité s'avère nécessaire. Un métro léger en site propre est inauguré en 1991 et son succès est immédiat : la ligne augmente la part modale des transports en commun de $43 \%$ en 1990 à $50 \%$ en 1992 (Bovy et Demierre, 2001). Elle arrive ensuite rapidement à saturation, la demande ayant été sous-estimée lors de sa conception. Sa capacité est améliorée avec le doublement du matériel roulant et l'augmentation des cadences. La construction d'une deuxième voie est actuellement à l'étude. Dès les années 2000, d'importants investissements dans la région lausannoise (deuxième ligne de métro, transformation des principales gares, etc.) renforcent attractivité des transports en commun, une tendance qui caractérise aussi les autres grandes villes suisses (Jemelin, 2008).

Ces mesures expliquent le niveau des parts modales (voir 4.1). Elles parviennent à juguler la croissance de la demande, mais nécessitent des investissements conséquents. L'amélioration des infrastructures peut également être à l'origine d'un phénomène de trafic induit en facilitant les déplacements. Ainsi, entre 2005 et 2017, la distance moyenne domicile-campus augmente de 13,8 à $16,2 \mathrm{~km}$. L'amélioration des infrastructures, combinée à la pénurie de logements dans la région lausannoise, a pu favoriser l'allongement des trajets pendulaires.

\subsection{Le campus régulé}

Le campus régulé renvoie à une démarche de gestion de la mobilité visant à modifier la demande. Outre des campagnes de promotion et l'instauration de services pour le vélo (stationnement, atelier, vélos en libre-service), ces mesures renvoient à la régulation du stationnement et à la définition des horaires de cours.

Jusqu'aux années 1990, le parking est gratuit, illimité et sans restriction d'accès. Une politique de stationnement est progressivement mise en place : autorisations selon le type d'utilisateurs (ex.: abonnement pour les seuls membres de la communauté universitaire), tarification et contrôles. L'Unil décide de ne pas augmenter le nombre de places de parking (1600) afin de favoriser les formes de mobilité durable et préserver les espaces verts. Le nombre d'abonnements de stationnement disponibles reste toutefois supérieur à la demande, ce qui tend à montrer que ces mesures ont suffi à dissuader certains automobilistes d'autant plus dans un contexte de moindre motorisation des jeunes (voir 4.2). 
Une autre mesure concerne les horaires des cours. En 2005, un tiers de la communauté universitaire rejoint le campus à $8 \mathrm{~h} 00$ (Figure 4). En 2006, le début des cours est décalé selon deux zones $(8 \mathrm{~h} 00 \text { et } 8 \mathrm{~h} \mathrm{30})^{10}$, ce qui permet de répartir les arrivées (moins de $20 \%$ des personnes arrivent à $8 \mathrm{~h}$ 00) (Figure 5). Cette désynchronisation, qui a surtout touché les étudiants, a permis de lisser les heures de pointe et d'accroître la capacité du métro le matin (un rattrapage a lieu à la pause de midi et les horaires sont identiques l'après-midi).

Figure 4. Taux d'arrivées (en jaune) et de départs (en bleu) sur le campus, en 2005

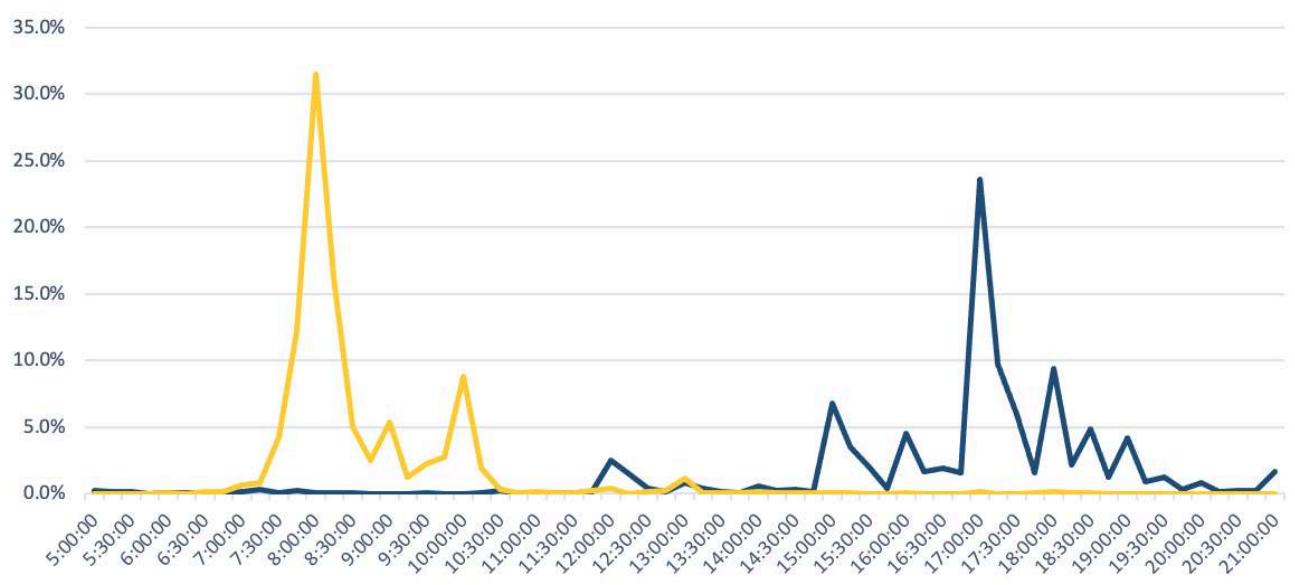

Figure 5. Taux d'arrivées (en jaune) et de départs (en bleu) sur le campus, en 2006

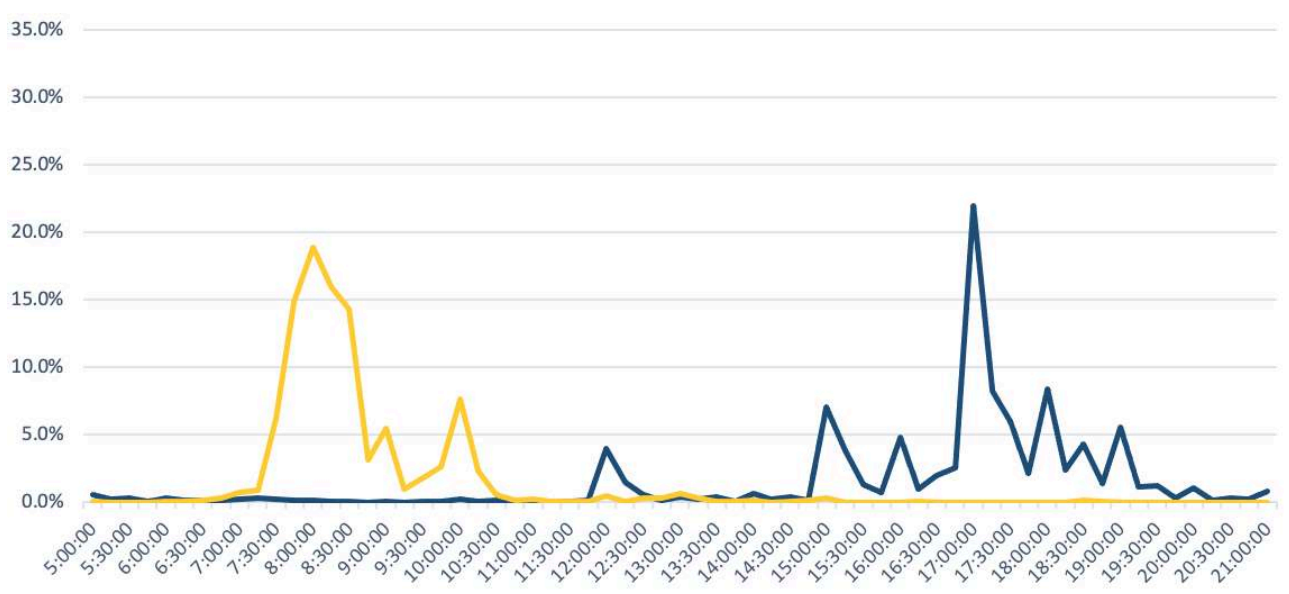

Ces mesures illustrent la marge de manœuvre dont dispose une université. Les conditions de stationnement ont une influence importante sur l'usage de la voiture et sont considérées, de manière générale, comme l'un des principaux leviers pour favoriser un report modal à la condition de proposer des alternatives et d'éviter le stationnement dans les quartiers environnants. Cette mesure peut toutefois se heurter à des questions d'acceptabilité sociale de la part des automobilistes. Le décalage des horaires de cours révèle la temporalité marquée des activités universitaires et, plus généralement, l'importance de l'organisation des temps urbains comme enjeu de l'aménagement du territoire (Ascher et Godard, 2003 ; Paquot, 2010). Son potentiel est toutefois limité par la croissance de la demande pour les transports publics dans le secteur de l'Unil. 


\subsection{Le campus intégré} mobilité, un de ces enjeux consiste à assurer l'interconnexion avec les quartiers environnants (passerelles, cheminements, etc.) pour les mobilités actives. En automne 2020, la commune qui accueille ce nouveau bâtiment (Chavannes-Près-Renens) a ainsi aménagé $7 \mathrm{~km}$ d'itinéraires cyclables sur son territoire. La question se pose également quant à l'organisation des circulations internes au campus. La planification originelle prévoyait une arrivée en voiture ou en transports en commun et des déplacements internes à pied, mais n'intégrait pas le vélo. La création d'itinéraires cyclables sécurisés et agréables est cruciale afin de tirer profit de la densification du campus.

\subsection{Le campus connecté}

52 La numérisation peut limiter la nécessité du présentiel et éviter certains déplacements à destination d'un campus connecté. C'est le cas du télétravail pratiqué surtout par les enseignants-chercheurs de manière informelle et sans qu'il soit possible de le quantifier avec les données disponibles ${ }^{11}$. Les propos relatifs à cette quatrième figure sont ainsi avant tout prospectifs dans un contexte où la crise de la Covid-19 a accéléré l'adoption à large échelle d'outils numériques pour l'enseignement et le travail à distance.

53 Ces pratiques pourraient contribuer à désynchroniser les flux, voire à les réduire. Associées à un enseignement moins axé sur les cours ex cathedra et davantage sur le travail personnel et de groupe, elles renforceraient l'importance de tiers lieux remplissant le rôle à la fois de bibliothèque, d'espace de partage et de travail, mais aussi de lieu de socialisation. Les employés quant à eux disposeraient d'une plus grande 
marge de manœuvre dans le lieu de réalisation de leurs tâches. L'université s'apparenterait ainsi de plus en plus à un campus nomade, à un lieu physique augmenté par la fluidité de réseaux virtuels. Le site de l'Unil renverrait à la fois à son campus et à unil.ch ; il serait simultanément territorialisé et hors sol (Maillard, 2013, 67).

Des limites à la numérisation sont toutefois à mentionner. Elles tiennent aux avantages du présentiel, notamment pour l'enseignement, mais aussi à des questions de faisabilité et de désirabilité (valorisation des contacts interpersonnels par exemple). Du point de vue de la gestion de la mobilité, le télétravail réduit le nombre de déplacements pendulaires. Il peut toutefois se concentrer sur certains jours de la semaine et s'accompagner d'effets rebonds (Ravalet et Rérat 2019). Pouvoir télétravailler pourrait représenter une prime à l'éloignement résidentiel, rendre les longs trajets pendulaires plus acceptables car moins fréquents, et faciliter la multirésidentialité, autant de tendances diminuant les bénéfices environnementaux du télétravail. Celle-ci serait d'autant plus probable que l'on assiste depuis 50 ans à une diminution des migrations interrégionales - soit entre cantons - en Suisse au profit de la pendularité de longue distance (Rérat, 2014 ; Schuler et al., 2007).

\section{Conclusion}

55 La mobilité constitue un enjeu de taille pour les universités qui drainent quotidiennement des flux importants de personnes. Cet article a pris l'Université de Lausanne comme étude de cas. Il a analysé la mobilité sous l'angle de la demande (les flux à destination du campus, leur évolution dans le temps ainsi que la variation des pratiques de mobilité des étudiants et collaborateurs selon leurs caractéristiques) et de l'offre (les politiques de mobilité ayant eu une influence sur le campus). Les principaux résultats apportent des éléments sur la manière de gérer la mobilité dans le cas des campus universitaires et appellent à des études comparatives.

En termes de parts modales, $60 \%$ des membres de l'Unil recourent uniquement aux transports en commun auxquels s'ajoutent la majeure partie des $10 \%$ qui pratiquent l'intermodalité et combinent les transports en commun avec un deux-roues (motorisé ou non) par exemple. Le vélo connaît une croissance appréciable (doublement de la part et triplement de l'effectif entre 2005 et 2017), alors que la voiture, voire les deuxroues motorisés sont en déclin. L'explication de ces tendances est à chercher dans les choix modaux des étudiants et du personnel et dans les différentes politiques de mobilité qui ont façonné le campus.

Des différences sont observées au sein de la communauté universitaire, quand bien même la destination, le campus, est identique. Elles se révèlent plus complexes qu'une simple opposition entre salariés et étudiants. On identifie le rôle de certaines caractéristiques sociodémographiques (genre, âge, statut professionnel, etc.), du contexte territorial (degré d'urbanité de la commune de résidence), des caractéristiques du déplacement (distance, fréquence) et de caractéristiques sociopsychologiques qui renvoient à la perception et à l'image des modes. On peut attribuer à ces dernières certaines différences quant à l'utilisation du vélo et de la voiture entre les tranches d'âge, les genres, mais aussi selon le statut et les facultés. Ce dernier résultat rappelle que le choix modal ne peut être réduit à un choix économiquement rationnel (De Witte et al., 2013). Le recours aux différents moyens de transport s'explique ainsi par l'articulation d'effets de genre, d'âge, de revenu, de contexte 
territorial (offre disponible et distance) et de valeurs et d'attitude (comme le reflètent notamment les différences entre facultés pour le vélo et la voiture). Cette diversité pourrait être prise en compte par des campagnes de communication modulables.

Nous avons proposé d'analyser les mesures de gestion de la mobilité par l'intermédiaire de quatre figures qui, si elles ne sont pas spécifiques au campus de l'Unil, pourraient être appliquées à d'autres cas d'étude. Le campus relié a consisté à développer une offre de transports publics desservant le campus. Le campus régulé a essentiellement impliqué la régulation du stationnement (qui a réduit l'attrait de la voiture) et le décalage du début des cours le matin (qui a lissé les heures de pointe pour les transports publics). Le campus intégré renvoie à la construction de logements dans un secteur qui n'accueillait que des bâtiments destinés à la recherche et à l'enseignement. L'importance des variables territoriales (type de commune et distance) apparaît par ailleurs clairement dans les logiques de choix modal. La proximité entre logements d'étudiants et lieux d'enseignement apparaît comme un moyen de valoriser les mobilités actives. Cette tendance est également à mettre en parallèle avec l'évolution des communes suburbaines et leur maturation en de véritables morceaux de ville (Dunham-Jones et Williamson, 2011). Finalement, le campus connecté se base sur la numérisation de l'enseignement, de la recherche et de l'administration qui pourrait réduire le nombre de déplacements et les répartir de manière plus équilibrée.

Ces quatre figures et leur articulation mettent en exergue certaines spécificités d'une université comme génératrice de mobilité. Si elle s'apparente à une petite ville par le nombre de ses étudiants et collaborateurs (la ville dans le sens de urbs), elle n'en a pas les compétences et prérogatives politiques (la ville dans le sens de civitas) et dépend par conséquent des collectivités territoriales qui l'entourent. Elle doit également répondre à une demande qui se distingue par des temporalités spécifiques (à l'échelle de la journée, de la semaine, des périodes de l'année) et dont une partie de la population (les étudiants) fait face à plusieurs contraintes (ressources financières limitées, vie chez les parents, etc.).

60 La présente étude comporte certaines limites. Les questions sur le choix modal dans l'enquête analysée sont d'ordre factuel et si le croisement avec le profil des personnes lève une partie du voile sur les différences au sein de la communauté universitaire, d'autres types de questions compléteraient de manière avantageuse cette démarche. C'est le cas de questions sur les raisons du choix modal des membres de la communauté universitaire (motivations et contraintes, image des différents modes, expérience et valorisation du temps de trajet, etc.), mais aussi des choix résidentiels (et les arbitrages entre déménagement et mobilité quotidienne) et de la pratique du télétravail (sa généralisation pendant les périodes les plus critiques de la crise sanitaire a provoqué un effet d'apprentissage inédit). Cet élargissement du questionnement permettrait de mieux comprendre la variation des pratiques entre individus et dans le temps.

61 Une autre limite dans l'optique de réduire l'empreinte écologique des universités est la non-prise en compte des mobilités professionnelles (colloques, conférences, jurys de thèse, travaux de terrain, etc.), alors que le milieu académique est très consommateur de ce type de mobilité souvent de longue distance et basée sur l'avion (Glover et al., 2017 ; Hopkins et al. , 2016). Ne pas agir sur cette mobilité pourrait annihiler les bénéfices environnementaux de la gestion de flux domicile-campus.

62 Un dernier enjeu crucial - tant en termes de politique que de recherche - renvoie au fait que les campus pourraient de plus en plus servir de living labs (König, 2013) dans la 
perspective de la transition vers une mobilité à basse empreinte carbone. C'est en particulier le cas lorsque des logements étudiants sont présents à proximité du campus. Les politiques mises en œuvre pourraient davantage prendre en compte le fait que la période des études constitue une phase cruciale dans l'apprentissage des mobilités durables, dont les effets pourraient perdurer dans les étapes ultérieures du parcours de vie.

\section{BIBLIOGRAPHIE}

Alamel A., 2015, An integrated Perspective of Student Housing Supply and Demand: Sustainability and Socio-Economic Differences, thèse de doctorat, géographie, Loughborough, University of Loughborough.

Ascher F., Godard F. (eds.), 2003, Modernité : la nouvelle carte du temps, La Tour d'Aigues, Éditions de l'Aube, Datar, collection « Monde en cours ».

Balsas C. J. L, 2003, « Sustainable transportation planning on college campuses », Transport Policy, vol. $10, \mathrm{n}^{\circ} 1, \mathrm{p} .35-49$.

Bourdin A., Campagnac É., 2014, «L'université : retour à la ville », Espaces et sociétés, vol. 159, n 4, p. 9-16, https://doi.org/10.3917/esp.159.0007.

Bovy P., Demierre J.-L., 2001, Du TSOL/Métro-Ouest 1991 au M1 de l'an 2000, Lausanne, Transports publics de la région lausannoise.

Brown J., Hess D. B., Shoup D., 2001, « Unlimited Access », Transportation, vol. 28, n 3, p. 233-267.

Cattaneo M., Malighetti P., Morlotti C., Paleari S., 2018, « Students' mobility attitudes and sustainable transport mode choice », International Journal of Sustainability in Higher Education, vol. $19, n^{\circ}$ 5, p. $942-962$.

Choplin A., Delage M., 2011, « Mobilités et espaces de vie des étudiants de l'Est francilien : des proximités et dépendances à négocier », Cybergeo, https://doi.org/10.4000/cybergeo.23840.

Coen L., Lambelet C. (eds.), 2011, L'Ouest pour horizon, Gollion, Infolio.

Courel J., Deguitre L., 2020, Les déterminants du choix modal. Synthèse des connaissances scientifiques, Paris, L'Institut Paris Région.

De Witte A., Hollevoet J., Dobruszkes F., Hubert M., Macharis C., 2013, « Linking modal choice to motility : A comprehensive review », Transportation Research Part A : Policy and Practice, vol. 49, p. 329-341.

Delmelle E. M., Delmelle E. C., 2012, « Exploring spatio-temporal commuting patterns in a university environment », Transport Policy, vol. 21, p. 1-9.

Dempsey N., Jenks M., 2010, « The Future of the Compact City », Built Environment, vol. 36, nº 1, p. 116-121.

Dunham-Jones E., Williamson J., 2011, Retrofitting suburbia : urban design solutions for redesigning suburbs, Hoboken, Wiley. 
Fürst E., 2014, « Making the way to the university environmentally sustainable : A segmentation approach », Transportation Research Part D : Transport and Environment, vol. 31, p. 1-12.

Genta C., Favaro S., Sonetti G., Barioglio C., Lombardi P., 2019, « Envisioning green solutions for reducing the ecological footprint of a university campus », International Journal of Sustainability in Higher Education, vol. 20, n 3, p. 423-440.

Glover A., Strengers Y., Lewis T., 2017, « The unsustainability of academic aeromobility in Australian universities », Sustainability : Science, Practice and Policy, vol. 13, n 1, p. 1-12.

Hopkins D., Higham J., Tapp S., Duncan T., 2016, « Academic mobility in the Anthropocene era : a comparative study of university policy at three New Zealand institutions ", Journal of Sustainable Tourism, vol. 24, $\mathrm{n}^{\circ}$ 3, p. 376-397.

Jarne A., Schuler M., Leyvraz J.-P., 2011, Mobilité des pendulaires EPFL -UNIL. Analyse spatialisée des résultats de l'enquête RUMBA, Lausanne, CEAT.

Jemelin C., 2008, Transports publics dans les villes : leur retour en force en Suisse, Lausanne, Presses polytechniques et universitaires romandes.

Kaufmann V., 2003, « Pratiques modales des déplacements de personnes en milieu urbain : des rationalités d'usage à la cohérence de l'action publique ", Revue d'économie régionale \& urbaine, 2003, n 1, p. 39, https://doi.org/10.3917/reru.031.0039.

Kim J., Schmöcker J.-D., Fujii S., 2016, « Exploring the relationship between undergraduate education and sustainable transport attitudes », International Journal of Sustainable Transportation, vol. $10, \mathrm{n}^{\circ} 4$, p. 385-392.

König A. (ed.), 2013, Regenerative sustainable development of universities and cities : the role of living laboratories, Cheltenham, Edward Elgar, $321 \mathrm{p}$.

Lachapelle U., Tanguay G. A., Neumark-Gaudet L., 2018, « Telecommuting and sustainable travel : Reduction of overall travel time, increases in non-motorised travel and congestion relief? », Urban Studies, vol. 55, $\mathrm{n}^{\circ}$ 10, p. 2226-2244.

Le Temps, 2016, « Les Suisses champions des kilomètres parcourus en train », 16 août.

Maillard N. (ed.), 2013, L'Université de Lausanne à Dorigny, Gollion, Infolio.

Melia S., Clark B., 2018, « What happens to travel behaviour when the right to park is removed?", Transport Policy, vol. 72, p. 242-247.

Miralles-Guasch C., Domene E., 2010, « Sustainable transport challenges in a suburban university : The case of the Autonomous University of Barcelona ", Transport Policy, vol. 17, n 6, p. 454-463.

OFS, 2014, L'espace à caractère urbain 2012, Neuchâtel, Office fédéral de la statistique.

Dell'Olio L., Cordera R., Ibeas A., Barreda R., Alonso B., Moura J. L., 2019, « A methodology based on parking policy to promote sustainable mobility in college campuses », Transport Policy, vol. 80, p. 148-156.

Paquot T., 2010, L'urbanisme c'est notre affaire!, Paris, Atalante.

Ravalet E., Rérat P., 2019, « Teleworking : Decreasing Mobility or Increasing Tolerance of Commuting Distances? ", Built Environment, vol. 45, n 4, p. 582-601.

Rérat P., 2014, « Highly qualified rural youth: Why do young graduates return to their home region? », Children's Geographies, vol. 12, n 1, p. 70-86. 
Rérat P., 2012, « Housing, the Compact City and Sustainable Development : Some Insights From Recent Urban Trends in Switzerland », International Journal of Housing Policy, vol. 12, $\mathrm{n}^{\circ} 2$,

p. 115-136.

Rérat P., Giacomel G., Martin A., 2019, Au travail à vélo... La pratique utilitaire de la bicyclette en Suisse, Neuchâtel, Éditions Alphil - Presses universitaires suisses.

Rérat P., Haldimann L., 2020, « Le permis de conduire chez les jeunes : qui ne le passe pas (encore) et pourquoi ? », Flux, nº 119-120, p. 5-24.

Rérat P., Vincent-Geslin S., Giacomel G., Martin A., Baehler D., 2016, « La baisse du permis de conduire chez les jeunes adultes : simple report ou désamour de la voiture ? ", Géo-Regards, n 9, p. $135-155$.

Rotaris L., Danielis R., 2015, « Commuting to college : The effectiveness and social efficiency of transportation demand management policies ", Transport Policy, vol. 44, p. 158-168.

Schuler M., Dessemontet P., Jemelin C., Jarne A., Pasche N., Haug W., 2007, Atlas des mutations spatiales de la Suisse, Zurich, Éditions Neue Zürcher Zeitung.

Shannon T., Giles-Corti B., Pikora T., Bulsara M., Shilton T., Bull F., 2006, « Active commuting in a university setting : Assessing commuting habits and potential for modal change », Transport Policy, vol. 13, n 3, p. 240-253.

Stead D., Banister D., 2001, « Influencing Mobility Outside Transport Policy », Innovation: The European Journal of Social Science Research, vol. 14, $n^{\circ} 4$, p. 315-330.

Tolley R., 1996, «Green campuses : cutting the environmental cost of commuting », Journal of Transport Geography, vol. 4, n 3, p. 213-217.

Vale D. S., Pereira M., Viana C. M., 2018, « Different destination, different commuting pattern? Analyzing the influence of the campus location on commuting ", Journal of Transport and Land Use, 5 vol. $11, \mathrm{n}^{\circ} 1$.

Van den Berg L., Russo A., 2004, The Student City : Strategic Planning for Student Communities in EU Cities, Aldershot, Ashgate.

Vu H. D., 2014, « Les grandes universités face aux enjeux de la production urbaine », Espaces et sociétés, vol. $159, \mathrm{n}^{\circ}$ 4, p. 17-35.

Weber M., 1922, Wirtschaft und Gesellschaft. Grundiss der verstehenden Soziologie, Mohr Siebeck, Tübingen.

\section{ANNEXES}

\section{Annexe 1. Répartition modale selon différentes variables explicatives}

\begin{tabular}{|l|l|l|l|l|l|l|l|l|}
\hline Variables & Modalités & \multicolumn{3}{|l|l|l|}{$\begin{array}{l}\text { Transports } \\
\text { publics }\end{array}$} & \multicolumn{2}{l|l}{$\begin{array}{l}\text { Mobilités } \\
\text { actives }\end{array}$} & \multicolumn{2}{l}{$\begin{array}{l}\text { Transports } \\
\text { individuels } \\
\text { motorisés }\end{array}$} \\
\hline & & 2005 & 2017 & 2005 & 2017 & 2005 & 2017 \\
\hline Sexe & Féminin & $64 \%$ & $71 \%$ & $6 \%$ & $10 \%$ & $30 \%$ & $19 \%$ \\
\hline
\end{tabular}




\begin{tabular}{|c|c|c|c|c|c|c|c|}
\hline & Masculin & $59 \%$ & $62 \%$ & $10 \%$ & $15 \%$ & $32 \%$ & $23 \%$ \\
\hline \multirow[t]{4}{*}{ Âge } & Jusqu'à 25 ans & $68 \%$ & $76 \%$ & $7 \%$ & $11 \%$ & $25 \%$ & $13 \%$ \\
\hline & 26-35 ans & $64 \%$ & $65 \%$ & $9 \%$ & $17 \%$ & $26 \%$ & $18 \%$ \\
\hline & $36-50$ ans & $32 \%$ & $45 \%$ & $5 \%$ & $11 \%$ & $63 \%$ & $44 \%$ \\
\hline & 51 ans et plus & $22 \%$ & $31 \%$ & $5 \%$ & $12 \%$ & $72 \%$ & $57 \%$ \\
\hline \multirow[t]{3}{*}{ Statut } & Étudiant & $67 \%$ & $74 \%$ & $8 \%$ & $12 \%$ & $42 \%$ & $23 \%$ \\
\hline & $\begin{array}{l}\text { Personnel administratif et } \\
\text { technique }\end{array}$ & $27 \%$ & $37 \%$ & $4 \%$ & $8 \%$ & $69 \%$ & $55 \%$ \\
\hline & Personnel académique & $48 \%$ & $54 \%$ & $11 \%$ & $20 \%$ & $42 \%$ & $26 \%$ \\
\hline \multirow[t]{9}{*}{ Faculté } & Administration centrale & $31 \%$ & $34 \%$ & $6 \%$ & $8 \%$ & $65 \%$ & $57 \%$ \\
\hline & Hautes études commerciales & $49 \%$ & $69 \%$ & $9 \%$ & $8 \%$ & $42 \%$ & $23 \%$ \\
\hline & Biologie et médecine & $62 \%$ & $64 \%$ & $11 \%$ & $16 \%$ & $28 \%$ & $21 \%$ \\
\hline & $\begin{array}{l}\text { Droit, sciences criminelles et } \\
\text { administration publique }\end{array}$ & $61 \%$ & $69 \%$ & $6 \%$ & $9 \%$ & $32 \%$ & $22 \%$ \\
\hline & $\begin{array}{l}\text { Théologie et sciences des } \\
\text { religions }\end{array}$ & $65 \%$ & $72 \%$ & $5 \%$ & $13 \%$ & $35 \%$ & $15 \%$ \\
\hline & $\begin{array}{l}\text { Géosciences et } \\
\text { environnement }\end{array}$ & $59 \%$ & $62 \%$ & $16 \%$ & $24 \%$ & $24 \%$ & $14 \%$ \\
\hline & Lettres & $70 \%$ & $73 \%$ & $7 \%$ & $14 \%$ & $22 \%$ & $13 \%$ \\
\hline & Sciences sociales et politiques & $68 \%$ & $71 \%$ & $6 \%$ & $12 \%$ & $26 \%$ & $18 \%$ \\
\hline & Institutions associées & $34 \%$ & $52 \%$ & $3 \%$ & $8 \%$ & $62 \%$ & $42 \%$ \\
\hline \multirow[t]{9}{*}{$\begin{array}{l}\text { Type de } \\
\text { commune }\end{array}$} & Grands centres & $77 \%$ & $75 \%$ & $7 \%$ & $15 \%$ & $16 \%$ & $11 \%$ \\
\hline & $\begin{array}{l}\text { Centres secondaires des } \\
\text { grands centres }\end{array}$ & $53 \%$ & $57 \%$ & $20 \%$ & $28 \%$ & $27 \%$ & $14 \%$ \\
\hline & Couronnes des grands centres & $41 \%$ & $58 \%$ & $10 \%$ & $13 \%$ & $48 \%$ & $28 \%$ \\
\hline & Centres moyens & $77 \%$ & $88 \%$ & $0 \%$ & $1 \%$ & $23 \%$ & $11 \%$ \\
\hline & $\begin{array}{l}\text { Couronnes des centres } \\
\text { moyens }\end{array}$ & $65 \%$ & $71 \%$ & $0 \%$ & $1 \%$ & $35 \%$ & $27 \%$ \\
\hline & Petits centres & $61 \%$ & $73 \%$ & $0 \%$ & $0 \%$ & $39 \%$ & $27 \%$ \\
\hline & $\begin{array}{l}\text { Communes rurales } \\
\text { périurbaines }\end{array}$ & $48 \%$ & $57 \%$ & $0 \%$ & $2 \%$ & $51 \%$ & $41 \%$ \\
\hline & Communes agricoles & $33 \%$ & $49 \%$ & $2 \%$ & $1 \%$ & $66 \%$ & $50 \%$ \\
\hline & Communes touristiques & $60 \%$ & $83 \%$ & $0 \%$ & $4 \%$ & $40 \%$ & $13 \%$ \\
\hline \multirow[t]{3}{*}{ Distance } & Moins de $5 \mathrm{~km}$ & $67 \%$ & $65 \%$ & $13 \%$ & $23 \%$ & $20 \%$ & $12 \%$ \\
\hline & $5-15 \mathrm{~km}$ & $46 \%$ & $61 \%$ & $3 \%$ & $7 \%$ & $51 \%$ & $32 \%$ \\
\hline & Plus de $15 \mathrm{~km}$ & $62 \%$ & $73 \%$ & $0 \%$ & $1 \%$ & $38 \%$ & $25 \%$ \\
\hline Fréquence & Jusqu'à 3 fois par semaine & $61 \%$ & $68 \%$ & $6 \%$ & $9 \%$ & $33 \%$ & $24 \%$ \\
\hline
\end{tabular}




\begin{tabular}{|l|l|l|l|l|l|l|l|}
\hline & 4 fois par semaine & $68 \%$ & $67 \%$ & $6 \%$ & $12 \%$ & $26 \%$ & $21 \%$ \\
\hline & 5 fois et plus par semaine & $60 \%$ & $66 \%$ & $9 \%$ & $14 \%$ & $32 \%$ & $20 \%$ \\
\hline
\end{tabular}

\section{NOTES}

1. La qualité du réseau ferroviaire est de manière générale à souligner, la Suisse occupant le premier rang mondial de la distance parcourue par personne en train (Le Temps, 2016).

2. Les répondants sélectionnent l'une de ces propositions : à pied, en transport public, à bicyclette, en voiture, en deux-roues motorisés, par un autre moyen, en utilisant plusieurs moyens. Des précisions sont demandées quant aux deux dernières modalités.

3. Les personnes utilisant une combinaison de moyens dans leurs trajets sont exclues de cette analyse.

4. Cette classe ne correspond qu'imparfaitement aux étudiants : certains d'entre eux sont plus âgés alors que des employés (notamment des services administratifs et techniques) en font partie.

5. Précisons que la moitié des étudiants de l'Unil ( $48 \%$ ) vivent chez leurs parents, un dixième dans un foyer d'étudiants et un tiers dans un logement indépendant (Fischer, 2015).

6. Sans distinction toutefois entre étudiants bachelor ou master.

7. Le même modèle général, testé séparément pour chaque année, montre une influence très similaire des différents paramètres.

8. Plus le résultat est éloigné de 1, plus l'impact de la variable est important. Il est donc possible de déterminer une hiérarchie entre les différents effets. En revanche, il n'est pas possible de définir un ordre de grandeur pour cet effet (il ne s'agit pas de probabilités simples). Ainsi, un rapport de probabilité de 2 ne signifie pas que la variable étudiée double la probabilité d'être mobile.

9. Le nombre de jours passés sur le campus n'a toutefois pas évolué entre 2005 et 2017.

10. Une troisième zone $(8 \mathrm{~h} 15)$ est instaurée dans l'École polytechnique fédérale voisine.

11. Le questionnaire renseigne sur les jours de présence sur le campus inférieur. Un nombre inférieur à cinq peut aussi indiquer un travail à temps partiel.

\section{RÉSUMÉS}

Les universités génèrent d'importants flux de mobilité quotidienne et les transports représentent pour elle un enjeu central tant en termes d'attractivité que d'empreinte écologique. Cet article, qui porte sur l'Université de Lausanne (Suisse), analyse les pratiques de mobilité des étudiants et du personnel (grâce à 13 enquêtes annuelles consécutives) et les principales logiques expliquant 
le recours aux différents moyens de transport. Il identifie ensuite quatre types d'intervention : le développement de l'offre de transport, la gestion de la demande, l'urbanisme et la numérisation. Dans l'ensemble, l'article apporte de nouvelles connaissances au sujet de la mobilité sur les campus universitaires et des leviers pour les transformer en communautés durables.

Universities generate important flows of commuting and transport represent for them a core challenges for their attractiveness and their ecological footprint. This paper addresses the case of the University of Lausanne, Switzerland. It analyses the mobility practices of students and staff (through a series of 13 annual surveys) and the main determinants that explain the use of the various means of transport. It then identifies four types of measures: the development of the transport supply, mobility management, planning and digitalization. On the whole the paper adds new knowledge to the issue of mobility on university campuses and the levers to make them into sustainable communities.

\section{INDEX}

Keywords : mobility, transport, university, campus, modal choice, sustainable mobility, policy Mots-clés : mobilité, transport, université, campus, choix modal, mobilité durable, politique

\section{AUTEUR}

\section{PATRICK RÉRAT}

Patrick Rérat est professeur de géographie des mobilités et codirecteur de l'observatoire universitaire du vélo et des mobilités actives à l'Université de Lausanne (Unil).

Institut de géographie et durabilité \& Observatoire universitaire du vélo et des mobilités actives. Patrick.rerat@unil.ch

Orcid : 0000-0001-6980-3336 